\title{
Cryoglobulinaemic vasculitis in light chain myeloma
}

\author{
Authors: Gwenno M Edwards and Abdulfattah Alejmi
}

KEYWORDS: cryoglobulinaemia, myeloma, hyperviscosity

DOI: $10.7861 /$ clinmed.2020-0880

\section{Case presentation}

A 79-year-old man with a history of treated lambda light chain myeloma in the previous year presented to the emergency department with a 2-week history of ischaemia of the hands and feet with livedo reticularis and a purpuric rash. He was admitted initially under the vascular team. Computed tomography angiography of his lower limbs was normal and he was managed conservatively with a prostaglandin analogue with little success. Within a day of being discharged by the vascular team, he was readmitted under the medical team with worsening peripheral ischaemia and multiple ulcers affecting his upper and lower limbs (Fig 1). On assessment he was drowsy and had myoclonus. His blood pressure was $107 / 88 \mathrm{mmHg}$ and his heart rate 106 beats per minute. He was apyrexial. Chest and abdominal examination were unremarkable.

He was found to have an acute kidney injury with a creatinine of $326 \mathrm{mmol} / \mathrm{L}$ from a baseline of $163 \mathrm{mmol} / \mathrm{L}$. His C-reactive protein (CRP) was $198 \mathrm{mg} / \mathrm{L}$.

\section{Diagnosis}

He was suspected of having a type I cryoglobulinaemic vasculitis secondary to a relapse of his light chain myeloma.

The inflammatory marker rise was thought to be due to an infected necrotic toe and the acute kidney injury as a result of the cryoglobulins.

The differential diagnoses for his altered consciousness level, myoclonus and confusion included uraemic encephalopathy and hyperviscosity syndrome.

The other potential diagnoses included anti-neutrophil cytoplasmic antibody-associated vasculitis, Sjogren syndrome and systemic lupus erythematosus.

\section{Initial management}

Despite intravenous fluids and antibiotics, his creatinine rose to $402 \mathrm{mmol} / \mathrm{L}$ and he became oligo-anuric and was therefore

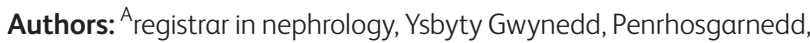
UK; ${ }^{B}$ consultant nephrologist, Ysbyty Gwynedd, Penrhosgarnedd, UK

\section{Fig 1. a) Acrocyanosis of fingers with ischaemia of left middle finger.} b) Multiple ulcers affecting the lower limbs.

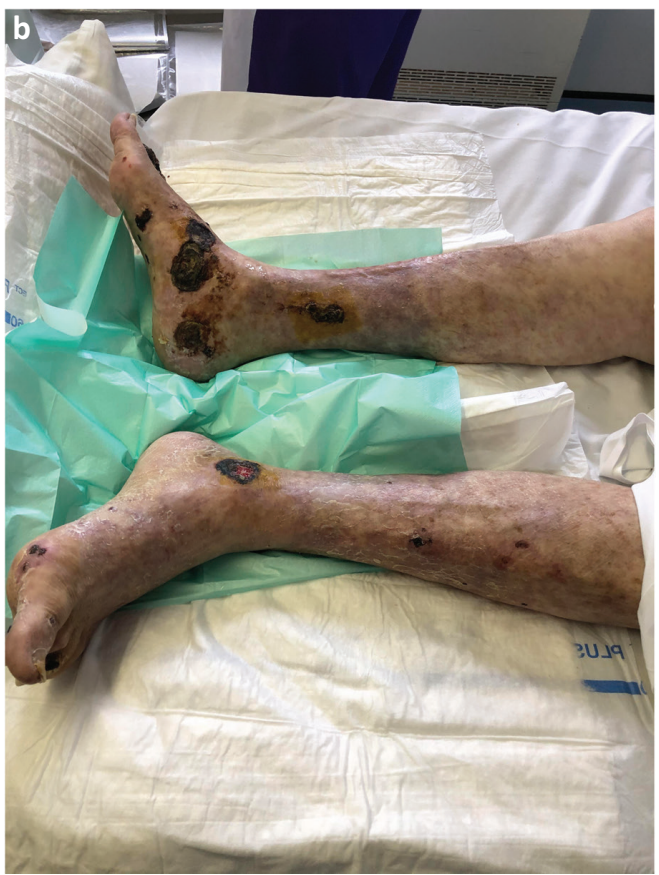


dialysed. Given his neurological manifestations, plasma exchange was started. We kept his temperature above $37.0^{\circ} \mathrm{C}$, including while on dialysis.

\section{Progression and outcome}

He was commenced on lenalidomide and dexamethasone by the haematology team for his multiple myeloma relapse.

Anti-nuclear antibodies and complement C3 and C4 were normal. His free light chain result was a lambda light chain level of 855.44 mg/L (baseline 63.33; normal range 5.7-26.3) with a kappa:lambda ratio of 0.015 (normal range $0.26-1.65$ ).

Cryoglobulin immunofixation showed the presence of an immunoglobulin $\mathrm{G}$ lambda monoclone consistent with a type I cryoglobulin.

The drowsiness and myoclonus improved with dialysis. With the initiation of plasma exchange and chemotherapy, the livedo reticularis and purpuric rash resolved, and the necrotic lesions improved dramatically. After 4 weeks, he was no longer dialysis dependent with his creatinine down to $156 \mathrm{mmol} / \mathrm{L}$. His CRP decreased to $<5 \mathrm{mg} / \mathrm{L}$. His repeat lambda light chain level was $51.41 \mathrm{mg} / \mathrm{L}$ (normal range 5.7-26.3) with a kappa:lambda ratio of 1.232 (normal range $0.26-1.65$ ).

\section{Discussion}

Cryoglobulinaemia is the presence of circulating immunoglobulins that precipitate in cold temperature and dissolve on rewarming. Type I cryoglobulinaemia is characterised by single monoclonal immunoglobulins, types II and III are mixed cryoglobulinaemias, with a monoclonal component in type II and only polyclonal components in type III. ${ }^{1}$

Type I cryoglobulinaemia is always related to a B-cell disorder, such as myeloma, chronic lymphocytic leukaemia and B-cell non-Hodgkin lymphoma. Mixed cryoglobulinaemia (types II and III) are associated with systemic autoimmune disease, lymphoproliferative disorders and chronic infection, especially hepatitis $C$ virus. ${ }^{1,2}$

Clinical manifestations are broad, including purpura, acrocyanosis, skin necrosis, skin ulceration, livedo reticularis, peripheral neuropathy and renal impairment. The most frequent histologic picture on renal biopsy is type I membranoproliferative glomerulonephritis with subendothelial deposits., ${ }^{1,2}$

Type I cryoglobulinaemia is considered a life-threatening disorder due to the severity of the cutaneous and renal involvement in addition to the underlying haematological disorder. Vascular occlusion is more frequent in type I cryoglobulinaemia and can be associated with hyperviscosity syndrome and coldinduced acrocyanosis. ${ }^{1}$

Currently there is little high-quality evidence for treatment due to the low incidence of type I cryoglobulinaemia. In patients with hyperviscosity syndrome, plasma exchange is indicated to remove the circulating immunoglobulins, however, treatment of the underlying plasma cell disorder is the mainstay of treatment. Patients should be advised to keep themselves warm to avoid precipitation of the cryoglobulins which will exacerbate acrocyanosis. $^{3,4}$

\section{Key points}

> Consider systemic disorders in a patient presenting with ischaemia affecting more than one limb.

$>$ Consider type I cryoglobulinaemia in a patient with an underlying B-cell disorder presenting with a purpuric rash or acrocyanosis.

> Type I cryoglobulinaemia carries a poor prognosis due to the underlying disorder and the severity of the presentation.

> Prompt diagnosis and treatment of the underlying B-cell disorder is vital.

> Hyperviscosity syndrome should be treated with plasma exchange.

\section{References}

1 Terrier B, Karras A, Kahn JE et al. The spectrum of type I cryoglobulinemia vasculitis new insights based on 64 cases. Medicine (Baltimore) 2013;92:61-8.

2 Ramos-Casals M, Stone JH, Cid MC, Bosch X. The cryoglobulinaemias. Lancet 2012:379:348-60.

3 Muchtar E, Magen H, Gertz MA. How I treat cryoglobulinemia. Blood 2017;129:289-98.

4 Rossa AD, Tavoni A, Bombardieri S. Hyperviscosity syndrome in cryoglobulinemia: clinical aspects and therapeutic considerations. Semin Thromb Hemost 2003;29:473-7.

Address for correspondence: Dr Gwenno Mair Edwards, Department of Nephrology, Ysbyty Gwynedd, Penrhosgarnedd, Bangor LL57 2PW, UK. Email: gwenno.edwards3@wales.nhs.uk 